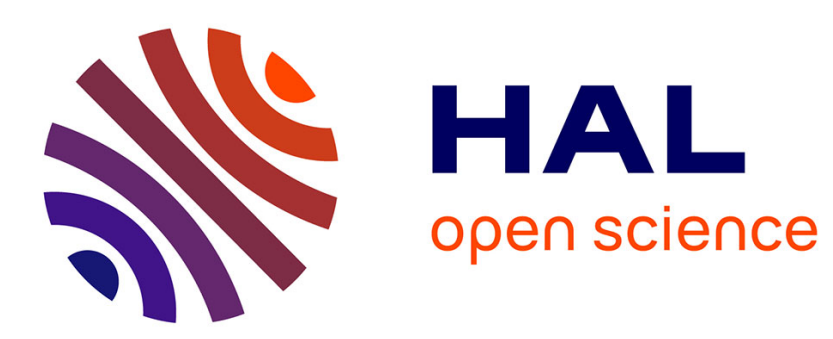

\title{
Vibrational calculation for the HOCO radical and the cis-HOCO anion
}

M. Mladenovic

\section{To cite this version:}

M. Mladenovic. Vibrational calculation for the HOCO radical and the cis-HOCO anion. Journal of Chemical Physics, 2012, 137 (1), pp.014306. hal-00750630

\section{HAL Id: hal-00750630 \\ https://hal.science/hal-00750630}

Submitted on 12 Nov 2012

HAL is a multi-disciplinary open access archive for the deposit and dissemination of scientific research documents, whether they are published or not. The documents may come from teaching and research institutions in France or abroad, or from public or private research centers.
L'archive ouverte pluridisciplinaire HAL, est destinée au dépôt et à la diffusion de documents scientifiques de niveau recherche, publiés ou non, émanant des établissements d'enseignement et de recherche français ou étrangers, des laboratoires publics ou privés. 


\section{AIP Chemicital Prysics}

\section{Vibrational calculation for the $\mathrm{HOCO}$ radical and the cis-HOCO anion}

Mirjana Mladenović

Citation: J. Chem. Phys. 137, 014306 (2012); doi: 10.1063/1.4732150

View online: http://dx.doi.org/10.1063/1.4732150

View Table of Contents: http://jcp.aip.org/resource/1/JCPSA6/v137/i1

Published by the American Institute of Physics.

Additional information on J. Chem. Phys.

Journal Homepage: http://jcp.aip.org/

Journal Information: http://jcp.aip.org/about/about_the_journal

Top downloads: http://jcp.aip.org/features/most_downloaded

Information for Authors: http://jcp.aip.org/authors

\section{ADVERTISEMENT}

\section{ACCELERATE COMPUTATIONAL CHEMISTRY BY 5X. TRY IT ON A FREE, REMOTELY-HOSTED CLUSTER. \\ NVIDIA.}




\title{
Vibrational calculation for the HOCO radical and the cis-HOCO anion
}

\author{
Mirjana Mladenovića) \\ Université Paris-Est, Laboratoire Modélisation et Simulation Multi-Echelle (MSME), UMR 8208 CNRS, \\ 5 bd Descartes, 77454 Marne la Vallée, France
}

(Received 8 May 2012; accepted 14 June 2012; published online 5 July 2012)

\begin{abstract}
We present numerically exact vibrational transitions for trans-HOCO, cis-HOCO, and cis- $\mathrm{HOCO}^{-}$ for the quartic force fields of Fortenberry et al. [J. Chem. Phys. 135, 134301 (2011); 135, 214303 (2011)], obtained by means of a computational strategy based on the discrete variable representation. Several adiabatic projection schemes have been employed to characterize the vibrational levels and to study the relevance of the intermode coupling (vibrational mixing). Our results help to clear up a large discrepancy between previously reported vibrational perturbation theory and vibrational configuration interaction predictions for the torsional frequency. (C) 2012 American Institute of Physics. [http://dx.doi.org/10.1063/1.4732150]
\end{abstract}

\section{INTRODUCTION}

As the key intermediate in the reaction between $\mathrm{HO}$ and $\mathrm{CO}$, the HOCO radical has received a great deal of attention over the past several decades from both the experimental and the theoretical side. Gas phase experiments were reported for the vibrational ground state of trans-HOCO and cis-HOCO, ${ }^{1-3}$ for the $v_{1}$ and $v_{2}$ fundamentals of trans$\mathrm{HOCO},{ }^{4,5}$ and for the low-frequency $v_{3}, v_{4}, v_{5}$ modes of the both isomers. ${ }^{6}$ The spectroscopic characterization of $\mathrm{HOCO}$ remains, thus, incomplete. On the other hand, most theoretical efforts have been devoted to the understanding of the unusual kinetic behaviour of the $\mathrm{HO}+\mathrm{CO}$ reaction. ${ }^{7}$ Several potential energy surfaces for HOCO were designed aiming at reaction dynamics studies, not intending to be accurate for vibrational spectroscopy interpretation. ${ }^{8-10}$ In other words, the theoretical characterization of realistic vibrating-rotating $\mathrm{HOCO}$ has remained poor until recently.

The most recent effort to provide a theoretical spectroscopic rationalization for HOCO is due to Fortenberry et al., who reported high quality coupled cluster separate quartic force fields for trans-HOCO (Ref. 11) and cis-HOCO. ${ }^{12}$ These force fields were derived at the complete basis set limit including core correlation and scalar relativistic effects, and high-order electron correlation terms for trans-HOCO. The force fields were employed to calculate the vibrational frequencies by means of second-order vibrational perturbation theory (VPT) and by a vibrational configuration interaction (VCI) approach. Whereas the VPT and VCI results reported there for the fundamental transitions of $v_{1}, v_{2}, v_{3}, v_{4}$, and $v_{5}$ agree within $1.5 \mathrm{~cm}^{-1}$ and $4.2 \mathrm{~cm}^{-1}$ for, respectively, trans and cis confomers, the VPT and VCI values for the torsional $v_{6}$ wavenumber differ by $12.6 \mathrm{~cm}^{-1}$ for trans-HOCO and by $26.3 \mathrm{~cm}^{-1}$ for $\mathrm{cis}$-HOCO. In the case of $\mathrm{cis}-\mathrm{HOCO}^{-}$, studied together with cis-HOCO in Ref. 12, the fundamental in-plane VPT and VCI transitions agree within $4.2 \mathrm{~cm}^{-1}$ and the VPT and VCI torsional $v_{6}$ wavenumbers within only $14.5 \mathrm{~cm}^{-1}$.

\footnotetext{
a)Electronic mail: mladenov@univ-mlv.fr.
}

The low level of agreement between the VPT and VCI results for the torsional $v_{6}$ mode found by Fortenberry et al. ${ }^{11,12}$ for trans-HOCO, cis-HOCO, and cis- $\mathrm{HOCO}^{-}$appears puzzling. In our previous study of HOCO (Ref. 13) using the potential energy surface of Schatz et al. ${ }^{8}$ vibrational states below the effective isomerization barrier were all found to reside in either the trans or the cis well (such that trans and cis levels could be identified in a rather simple manner by inspection of the expectation values of the torsional angle). In other words, the torsional motion of HOCO should not pose any major problem for the VPT approach, ${ }^{14}$ which is applicable in single minimum domains with small-amplitude vibrations. On the other hand, the torsional motion is intrinsically anharmonic (due to the trigonometric representation) and may undergo nonadiabatic mixing with the in-plane modes (as in the case of $\mathrm{H}_{2} \mathrm{O}_{2}$ (Ref. 15)). Compared to VPT, the VCI method ${ }^{16}$ is expected to better account for these effects. The influence of the anharmonicity and mode mixing is, however, less critical in low-energy domains (including the vicinity of the lowfrequency torsional fundamental of HOCO).

To conclusively clear up the situation regarding the VPT and VCI predictions for the torsional fundamental of trans-HOCO, cis-HOCO, and cis- $\mathrm{HOCO}^{-}$, accurate fulldimensional quantum mechanical calculations for the quartic force fields developed by Fortenberry et al. ${ }^{11,12}$ become indispensable.

\section{VIBRATIONAL STATE CALCULATIONS}

For the quartic force fields of Fortenberry et al., ${ }^{11,12}$ we carried out calculations for the vibrational energies of trans$\mathrm{HOCO}$, cis-HOCO, and cis- $\mathrm{HOCO}^{-}$by means of the discrete variable representation in an approach termed $\operatorname{DVR}(6) .{ }^{17}$ The computational strategy of the DVR(6) method uses a discretization of the entire six-dimensional angular-radial configuration space. The sequential inclusion of the internal degrees of freedom in combination with contraction schemes resulting from several diagonalization/truncation steps allows the construction of a final full-dimensional Hamiltonian 
matrix of relatively modest size. In the actual computations reported here, the primary basis sets included up to 4500 000 functions, whereas the size of the final six-dimensional Hamiltonian matrix was only up to 3000 .

The DVR(6) method is designed for the body-fixed formulation of the kinetic energy operator, expressed in terms of orthogonal internal coordinates. ${ }^{13}$ For $\mathrm{HO}_{a} \mathrm{CO}_{b}$, we use (diatom + diatom) coordinates $\left\{d_{1}, d_{2}, R, \theta_{1}, \theta_{2}, \chi\right\}$, where $d_{1}$ and $d_{2}$ refer to the $\mathrm{H}-\mathrm{O}_{a}$ and $\mathrm{C}-\mathrm{O}_{b}$ bond lengths, $R$ is the distance between the centers of mass of the $\mathrm{HO}_{a}$ and $\mathrm{CO}_{b}$ subunits, $\theta_{i}$ is the angle enclosed by the vectors $\mathbf{R}$ and $\mathbf{d}_{i}(i$ $=1,2$ ), and $\chi$ is the out-of-plane (torsional) angle. The orthogonal (diatom + diatom) coordinates are particularly well suited to describe the cis-trans isomerization of HOCO (torsional isomerization) and the dissociation of $\mathrm{HOCO}$ into the channel $\mathrm{HO}+\mathrm{CO}$.

The DVR(6) approach involves no re-expansion of the potential energy contribution and can use any potential energy representation. For $\mathrm{HOCO}$ and $c i s-\mathrm{HOCO}^{-}$, the quartic force fields in the common bond-distance-bond-angle coordinates subjected to a Morse-cosine transformation ${ }^{18}$ were employed, as recommended in Refs. 11 and 12. The DVR(6) methodology is appropriate for nonlinear tetratomic molecules, including systems with large amplitude torsional motion, as previously demonstrated for hydrogen peroxide. ${ }^{15}$ The actual computations for the separate quartic force fields of Fortenberry et al. ${ }^{11,12}$ for the HOCO isomers were carried out by truncating the Gauss-Chebyshev torsional DVR set, keeping the points distributed in $\chi<90^{\circ}$ and $\chi>90^{\circ}$ for the cis and trans confomers, respectively.

\section{RESULTS}

For the HOCO isomers, the vibrational modes $\left(v_{1}, v_{2}\right.$, $v_{3}, v_{4}, v_{5}, v_{6}$ ) refer approximately to symmetric HO stretch, $\mathrm{C}=\mathrm{O}$ stretch, $\mathrm{H}-\mathrm{O}-\mathrm{C}$ bend, $\mathrm{O}-\mathrm{C}$ stretch, $\mathrm{O}-\mathrm{C}-\mathrm{O}$ bend and torsion, respectively. For the cis anion, however, the $v_{4}$ mode is the $\mathrm{O}-\mathrm{C}-\mathrm{O}$ bend and $\nu_{5}$ is the $\mathrm{O}-\mathrm{C}$ stretch. This can be seen in Fig. 1, where the two-dimensional maps of the wavefunctions for the vibrational $v_{4}$ and $v_{5}$ states of cis-HOCO and $c i s-\mathrm{HOCO}^{-}$are displayed. The well-defined nodal patterns seen there for the two states of $c i s-\mathrm{HOCO}^{-}$clearly imply the excitation of the stretching motion in the $v_{5}$ state at $524.6 \mathrm{~cm}^{-1}$ and of the in-plane bending motion in the $v_{4}$ state at $669.4 \mathrm{~cm}^{-1}$. Consequently, the wavenumber of the stretching $\mathrm{C}-\mathrm{O}$ vibration of $c i s-\mathrm{HOCO}^{-}$is approximately two times smaller than the corresponding wavenumber of $1053 \mathrm{~cm}^{-1}$ for trans-HOCO and of $1046 \mathrm{~cm}^{-1}$ for cis-HOCO, due to a more shallow well in the direction of $r(\mathrm{C}-\mathrm{O})$. This is to be expected from the fact that the equilibrium value of $1.44 \AA$ for $r_{e}(\mathrm{C}-\mathrm{O})$ in $c i s-\mathrm{HOCO}^{-}$is found to be larger than $r_{e}(\mathrm{C}-\mathrm{O})$ of $1.34 \AA$ for trans-HOCO (Ref. 11) and of $1.33 \AA$ for $c i s-H O C O .{ }^{12}$ The bending $\mathrm{O}-\mathrm{C}-\mathrm{O}$ wavenumber of $c i s-\mathrm{HOCO}^{-}$is, on the other hand, larger by 52 and $68 \mathrm{~cm}^{-1}$ than the corresponding values of trans-HOCO and cis-HOCO, respectively. Note that the $v_{5}$ mode of $c i s-\mathrm{HOCO}^{-}$was previously attributed to the $\mathrm{O}-\mathrm{C}-\mathrm{O}$ bend and the $v_{4}$ mode to the $\mathrm{O}-\mathrm{C}$ stretch. ${ }^{12}$ The anharmonic $v_{4} / v_{5}$ mixing, seen in Fig. 1 for cis-HOCO, is explained later in the text.
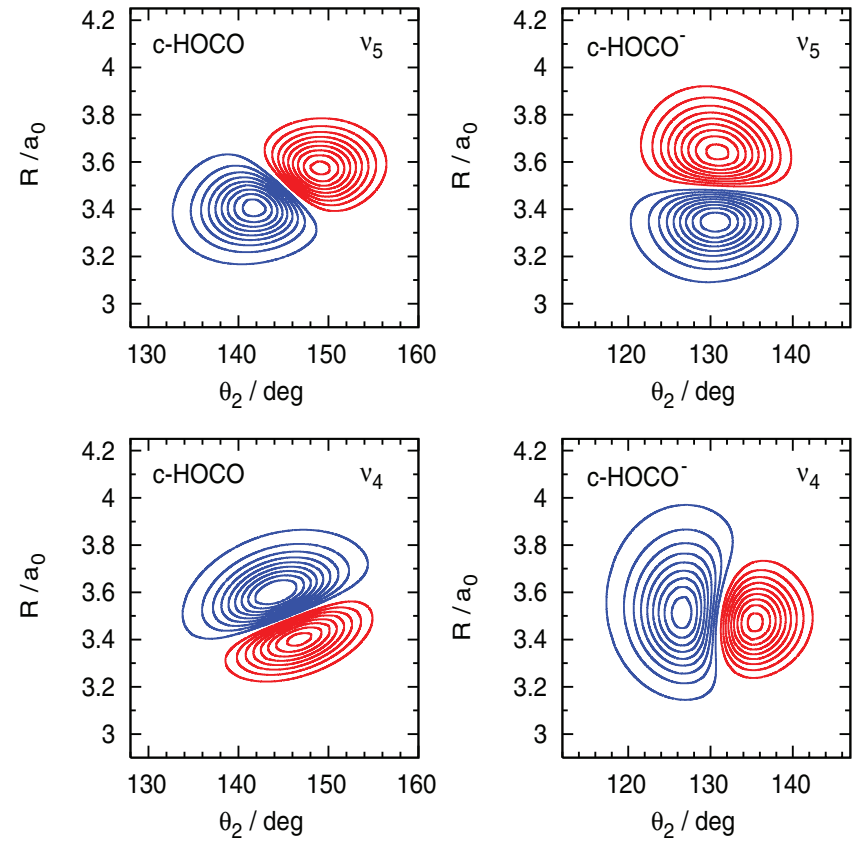

FIG. 1. Wavefunction contour maps for the vibrational states $v_{5}$ and $v_{4}$ of cis-HOCO (left) and of cis-HOCO${ }^{-}$(right). The remaining four coordinates are kept constant at their equilibrium values. The contours show where the wavefunction takes from $-95 \%$ to $95 \%$ with the step of $10 \%$ of its maximum value.

\section{A. Vibrational transitions}

Our results are summarized in Table I for trans-HOCO and $c i s-\mathrm{HOCO}$ and in Table II for $c i s-\mathrm{HOCO}^{-}$, together with the previous VPT and VCI values obtained for the same force fields. ${ }^{11,12}$ For trans-HOCO in Table I, we give the five-mode $\mathrm{VCI}$ energies denoted by $5 \mathrm{MR}-1$ in Ref. 11 . As readily seen in Tables I and II, the VPT torsional $v_{6}$ wavenumbers show good agreement within $1.3,3.6$, and $2.2 \mathrm{~cm}^{-1}$ with the DVR(6) values for, respectively, trans-HOCO, cis- $\mathrm{HOCO}$, and cis$\mathrm{HOCO}^{-}$. The corresponding deviations for the VCI $v_{6}$ results are, however, $11.3,22.7$, and $12.3 \mathrm{~cm}^{-1}$. In view of the relatively large deviations of the VCI torsional $v_{6}$ wavenumbers from the DVR(6) results, we may conclude that the VCI approach, compared to the VPT, appears less appropriate for the numerical treatment of the torsional motion of trans-HOCO, cis-HOCO, and cis- $\mathrm{HOCO}^{-}$. We may also note that Bowman et al. ${ }^{19}$ carried out vibrational configuration interaction calculations for the potential energy surface of Schatz et al. ${ }^{8}$ in the four-mode representation and obtained a torsional wavenumber of $671 \mathrm{~cm}^{-1}$ for trans-HOCO and of $422 \mathrm{~cm}^{-1}$ for cisHOCO. These $v_{6}$ values deviate by 7 and $11 \mathrm{~cm}^{-1}$ from the numerically exact DVR(6) results of 663.7 and $411.3 \mathrm{~cm}^{-1}$ reported previously. ${ }^{13}$

To gain insight into the intermode coupling and the torsional anharmonicity, Tables I and II also show the fivemode (stretch-bend) energies $\varepsilon^{(5 D)}$ and the effective torsional wavenumbers $v_{6}^{\text {eff }}$ from our calculations. The energies $\varepsilon^{(5 D)}$ were obtained for the torsional angle fixed at the respective equilibrium values, thus, neglecting the torsional kinetic contribution. The effective torsional $v_{6}^{\text {eff }}\left(v_{i}\right)$ wavenumbers upon the excitation of the mode $v_{i}$ were determined from the 
TABLE I. Fundamental vibrational transitions and zero-point energy (ZPE) of HOCO (in $\mathrm{cm}^{-1}$ ). In addition to the full-dimensional DVR(6) vibrational energies $E^{(6 D)}$, the in-plane five-mode energies $\varepsilon^{(5 D)}$ calculated for the planar configuration and the effective torsional wavenumbers $v_{6}^{\text {eff }}$ determined according to Eq. (1) are also shown.

\begin{tabular}{|c|c|c|c|c|c|c|c|c|c|c|}
\hline & \multicolumn{5}{|c|}{ trans-HOCO } & \multicolumn{5}{|c|}{ cis-HOCO } \\
\hline & \multicolumn{3}{|c|}{ This work } & \multicolumn{2}{|c|}{ Ref. 11} & \multicolumn{3}{|c|}{ This work } & \multicolumn{2}{|c|}{ Ref. 12} \\
\hline & $\varepsilon^{(5 D)}$ & $E^{(6 D)}$ & $v_{6}^{e f f}$ & VPT & $\mathrm{VCI}$ & $\varepsilon^{(5 D)}$ & $E^{(6 D)}$ & $v_{6}^{e f f}$ & VPT & $\mathrm{VCI}$ \\
\hline$v_{1}$ & 3648 & 3642.2 & 490 & 3642.0 & 3640.5 & 3448 & 3450.4 & 565 & 3450.8 & 3452.3 \\
\hline$v_{2}$ & 1862 & 1861.0 & 497 & 1861.0 & 1861.1 & 1823 & 1824.3 & 563 & 1823.4 & 1824.1 \\
\hline$v_{3}$ & 1218 & 1217.2 & 497 & 1217.3 & 1218.0 & 1273 & 1282.1 & 572 & 1284.4 & 1280.2 \\
\hline$v_{4}$ & 1054 & 1052.7 & 495 & 1053.0 & 1052.8 & 1049 & 1045.6 & 547 & 1045.9 & 1042.4 \\
\hline$v_{5}$ & 615 & 617.2 & 504 & 617.3 & 617.5 & 599 & 601.3 & 567 & 601.7 & 601.2 \\
\hline$v_{6}$ & & 499.9 & 467 & 501.2 & 488.6 & & 562.9 & 536 & 566.5 & 540.2 \\
\hline ZPE & & 4556.6 & & 4560.9 & 4551.4 & & 4492.7 & & 4491.4 & 4485.7 \\
\hline
\end{tabular}

full-dimensional vibrational energies $E^{(6 D)}$ as

$$
v_{6}^{e f f}\left(v_{i}\right)=E_{v_{i}+v_{6}}^{(6 D)}-E_{v_{i}}^{(6 D)}
$$

Note that the vibrational levels $v_{i}$ and $v_{i}+v_{6}$ are of different parity.

The coupling between the torsion (out-of-plane motion) and the in-plane vibrations appears to be weak for most of the vibrational modes in $\mathrm{HOCO}$ and $c i s-\mathrm{HOCO}^{-}$, as seen by the small differences between the five-mode energies $\varepsilon^{(5 D)}$ and the accurate level energies $E^{(6 D)}$. However, there are several exceptions. To study separability of the torsion and in-plane modes, we used the adiabatic projection method involving zero-order (adiabatic) basis functions constructed by means of the adiabatic torsion approximation following the quantum state character correlation scheme. ${ }^{17}$ The zero-order function denoted by $\mid i, \alpha ; p)$ describes the adiabatic level with $i$ quanta in the five-mode in-plane vibration and $\alpha$ quanta in the torsion for a given parity $p$.

In Tables I and II, the largest differences between $\varepsilon^{(5 D)}$ and $E^{(6 D)}$ are for the $v_{3}$ level of cis-HOCO $\left(9 \mathrm{~cm}^{-1}\right)$ and for the $v_{3}$ level of $c i s-\mathrm{HOCO}^{-}\left(25 \mathrm{~cm}^{-1}\right)$. These are caused by the zero-order $v_{3} / 2 v_{6}$ mixing, as evident from the adiabatic expansions obtained in the DVR(6) calculations for cis-HOCO (5\% mixing)

$$
\begin{aligned}
\left|v_{3}\right\rangle & =-\underline{0.97} \mid 4,0 ; 0)+0.23 \mid 0,1 ; 0), \\
\left|2 v_{6}\right\rangle & =0.23 \mid 4,0 ; 0)+\underline{0.96} \mid 0,1 ; 0),
\end{aligned}
$$

\begin{tabular}{|c|c|c|c|c|c|}
\hline \multirow[b]{2}{*}{ Mode } & \multicolumn{3}{|c|}{ This work } & \multicolumn{2}{|c|}{ Ref. 12} \\
\hline & $\varepsilon^{5 D}$ & $E^{(6 D)}$ & $v_{6}^{e f f}$ & VPT & VCI \\
\hline$v_{1}$ & 3296 & 3306.0 & 631 & 3306.4 & 3306.5 \\
\hline$v_{2}$ & 1565 & 1564.6 & 616 & 1569.4 & 1565.2 \\
\hline$v_{3}$ & 1149 & 1124.3 & 664 & 1128.1 & 1125.0 \\
\hline$v_{4}$ & 675 & 669.4 & 600 & 669.8 & 669.9 \\
\hline$v_{5}$ & 537 & 524.6 & 580 & 526.4 & 524.6 \\
\hline$v_{6}$ & & 610.2 & 592 & 612.5 & 598.0 \\
\hline ZPE & & 4063.6 & & 4066.7 & 4058.6 \\
\hline
\end{tabular}

TABLE II. Same as Table I, only for $c i s-\mathrm{HOCO}^{-}$. and for $c i s-\mathrm{HOCO}^{-}(24 \%$ mixing $)$

$$
\begin{aligned}
\left|v_{3}\right\rangle & =\underline{0.86} \mid 4,0 ; 0)-0.49 \mid 0,1 ; 0), \\
\left|2 v_{6}\right\rangle & =0.50 \mid 4,0 ; 0)+\underline{0.85} \mid 0,1 ; 0),
\end{aligned}
$$

where $\left.\mid 0,1 ; 0)=\mid 2 v_{6}\right)$ and $\left.\left.\mid 4,0 ; 0\right)=\mid v_{3}\right)$ for even parity $(p=0)$. The latter two-component expansions provide more than $95 \%$ of the full-dimensional wavefunction $|v\rangle$ for the state $v$. The accurate level $2 v_{6}$ at $1099 \mathrm{~cm}^{-1}$ is below $v_{3}$ for cis-HOCO, whereas the $2 v_{6}$ level of cis- $\mathrm{HOCO}^{-}$at 1202 $\mathrm{cm}^{-1}$ is above the corresponding $v_{3}$ level.

In order to understand the origin of the discrepancies between our converged variational results and the VPT and VCI results for the same potential energy surface we decided to investigate the relative importance of several key coupling mechanisms. If this effect is exclusively due to the kinetic derivative coupling involving the torsion was examined by full-dimensional calculations carried out by omitting the corresponding contributions from the kinetic energy operator [terms (d), (e), and (f) of Eq. (4) in Ref. 17]. The latter omission of kinetic energy terms affects primarily the bending $v_{3}$ and torsional $v_{6}$ wavenumbers, found to be changed, respectively, by $-0.6,-2.1$, and $+2.5 \mathrm{~cm}^{-1}$ and by $+6.0,-1.5$, and $+0.7 \mathrm{~cm}^{-1}$ for trans-HOCO, cis-HOCO, and cis- $\mathrm{HOCO}^{-}$, whereby the changes of the other fundamentals were smaller than $0.5 \mathrm{~cm}^{-1}$.

The effective torsional wavenumber $v_{6}^{\text {eff }}$ provides further clues concerning the nature of coupling effects in various energy domains. This follows from the fact that effective (adiabatic) torsional profiles, guiding the torsion for a given state of the in-plane vibrations, depend on the latter state due to a non-uniform variation of the effective wavenumbers of the in-plane modes with the torsional angle. The torsional motion is highly anharmonic, such that torsional overtones may perturb different zero-order stretch-bend states in different energy regions, thus, leading to energy-dependent mixing patterns.

The adiabatic torsional wavenumbers, calculated to be 508.3, 566.2, and $622.1 \mathrm{~cm}^{-1}$, are $+8.4,+3.3$, and +11.9 $\mathrm{cm}^{-1}$ larger than the full-dimensional $v_{6}$ results, indicating, thus, that the nonadiabatic effects are most important for cis$\mathrm{HOCO}^{-}$and least important for cis-HOCO. The torsional motion appears more anharmonic for trans-HOCO than for 
cis-HOCO and cis- $\mathrm{HOCO}^{-}$. We see this with the help of $v_{6}^{\text {eff }}\left(v_{6}\right)$, which is by 33,27 , and $18 \mathrm{~cm}^{-1}$ lower than the torsional fundamental for, respectively, trans-HOCO, cis$\mathrm{HOCO}$, and $c i s-\mathrm{HOCO}^{-}$. Upon excitation of the in-plane modes, $v_{6}^{\text {eff }}\left(v_{3}\right)$ and $v_{6}^{\text {eff }}\left(v_{4}\right)$ for cis-HOCO and $v_{6}^{\text {eff }}\left(v_{1}\right)$ for trans-HOCO are changed most prominently with respect to $v_{6}$ in Table I. The lowering even by $16 \mathrm{~cm}^{-1}$ of $v_{6}^{\text {eff }}\left(v_{4}\right)$ for cis-HOCO is, for instance, easy to understand in terms of the zero-order odd-parity $v_{4}+v_{6} / 3 v_{6}$ mixing, described by the following adiabatic expansions:

$$
\begin{aligned}
\left|v_{4}+v_{6}\right\rangle & =\underline{0.77} \mid 2,0 ; 1)-0.61 \mid 0,1 ; 1), \\
\left|3 v_{6}\right\rangle & =0.64 \mid 2,0 ; 1)-\underline{0.71} \mid 0,1 ; 1) .
\end{aligned}
$$

Due to the latter mode mixing, the zero-order state $\mid v_{4}$ $\left.\left.+v_{6}\right)=\mid 2,0 ; 1\right)$ is pushed down by the perturbing state $\left.\left|3 v_{6}\right|=\mid 0,1 ; 1\right)$, resulting in $v_{6}^{\text {eff }}\left(v_{3}\right)$ smaller than $v_{6}$. The mode mixing, as those in Eqs. (2)-(4), becomes more extensive for higher torsional excitations. Whereas the mixing in order of $24 \%$ is seen for $v_{3} / 2 v_{6}$ in Eq. (3), the mixing $v_{3}$ $+v_{6} / 3 v_{6}$ for $c i s-\mathrm{HOCO}^{-}$is found to amount to $34 \%$, causing an increase of $v_{6}^{\text {eff }}\left(v_{3}\right)$ by $54 \mathrm{~cm}^{-1}$ relative to $v_{6}$, as seen in Table II.

The full-dimensional wavefunctions of Fig. 1 show the $v_{4} / v_{5}$ mixing in cis-HOCO. To characterize the in-plane vibration, we employ the zero-order functions obtained in the adiabatic bend approximation for a fixed (e.g., equilibrium) value of the torsional angle. The adiabatic five-mode wavefunctions $|k| \gamma)$ are, thus, specified by the $k$ th state of the three-dimensional stretching vibration and the $\gamma$ th state of the two-dimensional bending vibration. ${ }^{13}$ The adiabatic expansions with respect to $|k| \gamma$ ) for the $v_{4}$ and $v_{5}$ states of the planar $c i s-\mathrm{HOCO}[\chi=0]$ are given by

$$
\begin{aligned}
\left|\nu_{5}\right\rangle_{[\chi=0]}= & -\underline{0.91} \mid k=0) \mid \gamma=1) \\
& -0.38 \mid k=1) \mid \gamma=0), \\
\left|\nu_{4}\right\rangle_{[\chi=0]}= & -0.37 \mid k=0) \mid \gamma=1) \\
& +\underline{0.85} \mid k=1) \mid \gamma=0) .
\end{aligned}
$$

For planar cis- $\mathrm{HOCO}^{-}[\chi=0]$, we have

$$
\begin{aligned}
& \left.\left.\left|\nu_{5}\right\rangle_{[\chi=0]}=-0.21 \mid k=0\right) \mid \gamma=1\right) \\
& +\underline{0.92} \mid k=1) \mid \gamma=0) \\
& +0.25 \mid k=0) \mid \gamma=2) \text {, } \\
& \left.\left.\left|v_{4}\right\rangle_{[\chi=0]}=-\underline{0.96} \mid k=0\right) \mid \gamma=1\right) \\
& -0.18 \mid k=1) \mid \gamma=0) \text {, }
\end{aligned}
$$

where $\mid k=0) \mid \gamma=2$ ) stands for the adiabatic $\nu_{3}$ state. The zero-order origin of the vibrational levels in Eqs. (5) and (6) is easy to identify since the dominant zero-order contributions amount to at least $72 \%$. Note, however, the different zero-order level origins for the $v_{4}$ and $v_{5}$ states of cis-HOCO and $c i s-\mathrm{HOCO}^{-}$. The corresponding five-mode energies are denoted by $\varepsilon^{(5 D)}$ in Tables I and II.

\section{B. Additional test calculations}

To access the coupling effects in $\mathrm{HOCO}$ and cis- $\mathrm{HOCO}^{-}$ by other means, several additional calculations were carried out.

Choosing a single potential adapted discrete point per radial degree of freedom, the torsional wavenumbers were calculated to be $501.8,562.3$, and $620.9 \mathrm{~cm}^{-1}$, deviating, thus, by $+1.9,-0.6$, and $+10.6 \mathrm{~cm}^{-1}$ from the full-dimensional results for, respectively, trans-HOCO, cis-HOCO, and cis$\mathrm{HOCO}^{-}$. A single discrete point was subsequently used also for the bending coordinates, such that the basis set size was equal to the size of the torsional base and in order of 10 . We obtained torsional fundamentals of 517.6, 561.1, and 621.3 $\mathrm{cm}^{-1}$, which differ by $+17.7,-1.8$, and $+11.0 \mathrm{~cm}^{-1}$ from the exact $v_{6}$ values. The reduced size calculations clearly show that the torsional-bending coupling is important for trans$\mathrm{HOCO}$ and the torsional-radial coupling for $c i s-\mathrm{HOCO}^{-}$. At the same time, $v_{6}$ of $c i s-\mathrm{HOCO}$ appears rather insensitive to the dramatic reduction of the stretch-bend base.

Especially interesting were the results of calculations carried out for modified force fields. Using only the quadratic force fields, we calculated torsional $v_{6}$ wavenumbers of 512.8, 545.9 , and $628.0 \mathrm{~cm}^{-1}$. Neglect of the quartic torsional contribution arising from the term $f_{6666}$ gave $v_{6}$ of 501.2, 543.5, and $613.0 \mathrm{~cm}^{-1}$ for, respectively, trans-HOCO, cis-HOCO, and $c i s-\mathrm{HOCO}^{-}$. The potential energy effect due to the $f_{6666}$ part (the anharmonicity effect) is, thus, crucial for the accurate determination of $v_{6}$ in $\mathrm{cis}$-HOCO. One should note that the latter two $v_{6}$ values of 545.9 and $543.5 \mathrm{~cm}^{-1}$ for cis-HOCO are both in very close agreement with the corresponding VCI result of $540.2 \mathrm{~cm}^{-1}$ listed in Table I.

Morse-cosine transformed quartic force fields for trans-HOCO, cis-HOCO, and cis- $\mathrm{HOCO}^{-}$were used in our DVR(6) calculations, as well in the previous VCI computations. ${ }^{11,12}$ In the Morse-cosine description based on sine functions for the torsion, $f_{6666}$ of +0.303 for cis-HOCO is of different sign and much larger than $f_{6666}$ of -0.019 for trans-HOCO and $f_{6666}$ of -0.077 for cis- $\mathrm{HOCO}^{-}$. The quartic force field constants in the ordinary internal coordinates are found in Table V of Ref. 11 and Tables VII and VIII of Ref. 12. As seen there, the magnitude of the $F_{6666}$ coefficient of -0.13 for cis-HOCO in the simple bond-distance-bond-angle representation is smaller than $F_{6666}$ of -0.49 for trans-HOCO and $F_{6666}$ of -0.79 for $c i s-\mathrm{HOCO}^{-}$. Note that the large positive $f_{6666}$ constant for cis-HOCO and small negative $f_{6666}$ for trans-HOCO and cis- $\mathrm{HOCO}^{-}$in the Morse-cosine description stem from the coordinate transformation term, involving the bond-distance-bond-angle constants $F_{66}$ and $F_{6666}$ of different relationship for these three systems.

For the quartic force fields expressed in terms of the bond-distance-bond-angle coordinates, the torsional $v_{6}$ wavenumbers were calculated to be $496.7,566.6$, and 607.5 $\mathrm{cm}^{-1}$ for, respectively, trans-HOCO, cis-HOCO, and cis$\mathrm{HOCO}^{-}$. These values differ by $+3.2,+3.7$, and -2.7 
$\mathrm{cm}^{-1}$ from the corresponding $v_{6}$ values in Tables I and II, obtained for the Morse-cosine transformed quartic force fields.

\section{CONCLUSION}

High-quality $a b$ initio force fields may provide reference data useful for experimental measurements, including identification of unknown species. ${ }^{20} \mathrm{In}$ addition to equilibrium rotational constants and harmonic vibrational frequencies, which routinely accompany the electronic structure analysis, the evaluation of accurate force fields beyond the quadratic formulation is necessary for generating data to support/assist detailed spectroscopic studies.

The torsional motion in more-than-three particle systems may pose an obstacle to theoretical methods for the rovibrational energy determination. In addition to the potential energy coupling with the other vibrational modes, the torsion is also under the influence of global kinetic energy coupling. This can be seen from the torsional reduced mass, whose inverse in terms of the orthogonal coordinates $\left\{d_{1}, d_{2}, R, \theta_{1}, \theta_{2}\right.$, $\chi\}$ is given for tetratomic molecules by ${ }^{17}$

$$
\begin{aligned}
F\left(R, d_{1}, d_{2}, \theta_{1}, \theta_{2}\right)= & {\left[f\left(d_{1}, R\right) / \sin ^{2} \theta_{1}\right.} \\
& \left.+f\left(d_{2}, R\right) / \sin ^{2} \theta_{2}-2 / \mu_{R} R^{2}\right],
\end{aligned}
$$

where $f\left(d_{i}, R\right)=1 / \mu_{i} d_{i}^{2}+1 / \mu_{R} R^{2}$, whereas $\mu_{1}, \mu_{2}, \mu_{R}$ are the reduced masses attributed to the radial degrees of freedom. The accurate treatment of the term involving $F\left(R, d_{1}\right.$, $\left.d_{2}, \theta_{1}, \theta_{2}\right)$ is indispensable whenever real vibrating molecules explore close-to-linearity arrangements (quasi-linear and linear molecular systems). ${ }^{21}$ For molecules with high barriers to linearity, on the other hand, an approximate treatment of the angular singularities in Eq. (7) may be sufficient. Torsion is an out-of-plane motion, possessing intrinsic rotational character, such that it may additionally pose a problem to approaches based on rectilinear normal coordinates.

In the present work, we carried out the vibrational analysis for trans-HOCO, cis-HOCO, and cis- $\mathrm{HOCO}^{-}$, employing the quartic force fields recently developed by Fortenberry et al. ${ }^{11,12}$ with the purpose to clear up the discrepancy found by these authors between the VPT and VCI results for the torsional fundamental. Another purpose of this work was to study the intermode coupling, providing a convenient way to hint at possible causes for the emergence of the VPT-VCI differences. We accordingly used a numerically exact quantum mechanical method involving no dynamical approximations and no re-expansion of the potential energy contribution. Our data show that the fundamental transitions obtained by means of vibrational second-order perturbation theory are more trustworthy with respect to vibrational configuration interaction predictions in the case of trans-HOCO, $c i s-\mathrm{HOCO}$, and $c i s-\mathrm{HOCO}^{-}$.

Our final remark is regarding the existence of two planar equilibrium structures of $\mathrm{HOCO}$, with the cis isomer being $611 \mathrm{~cm}^{-1}$ higher in energy than the trans form. ${ }^{22}$ The torsional motion of HOCO is given by a double-minimum anharmonic potential with a non-planar saddle point lying $\sim 3268$ $\mathrm{cm}^{-1}$ above the trans minimum. ${ }^{23}$ To gain insight into the torsional structure of the vibrating and rotating HOCO radical, a common potential energy representation for the two $\mathrm{HOCO}$ forms is, however, required. This is a part of our work currently in progress.

\section{ACKNOWLEDGMENTS}

The author thanks Dr. X. Huang and Dr. T. J. Lee for sending her the quartic force field for trans-HOCO. Professor Marius Lewerenz is thanked for many helpful discussions.

${ }^{1}$ H. E. Radford, W. Wei, and T. J. Sears, J. Chem. Phys. 97, 3989 (1992).

${ }^{2}$ T. J. Sears, H. E. Radford, and M. A. Moore, J. Chem. Phys. 98, 6624 (1993).

${ }^{3}$ T. Oyama, W. Funato, Y. Sumiyoshi, and Y. Endo, J. Chem. Phys. 134, 174303 (2011).

${ }^{4}$ T. J. Sears, W. M. Fawzy, and P. M. Johnson, J. Chem. Phys. 97, 3996 (1992).

${ }^{5}$ J. Petty and C. B. Moore, J. Mol. Spectrosc. 161, 149 (1993).

${ }^{6}$ C. J. Johnson, M. E. Harding, B. L. J. Poad, J. F. Stanton, and R. E. Continetti, J. Am. Chem. Soc. 133, 19606 (2011).

${ }^{7}$ H. Guo, Int. Rev. Phys. Chem. 31, 1 (2012).

${ }^{8}$ G. C. Schatz, M. S. Fitzcharles, and L. B. Harding, Faraday Discuss. 84, 359 (1987).

${ }^{9}$ M. J. Lakin, D. Troya, G. C. Schatz, and L. H. Harding, J. Chem. Phys. 119, 5848 (2003).

${ }^{10}$ J. Li, Y. Wang, B. Jiang, J. Ma, R. Dawes, D. Xie, J. M. Bowman, and H. Guo, J. Chem. Phys. 136, 041103 (2012).

${ }^{11}$ R. C. Fortenberry, X. Huang, J. S. Francisco, T. D. Crawford, and T. J. Lee, J. Chem. Phys. 135, 134301 (2011).

${ }^{12}$ R. C. Fortenberry, X. Huang, J. S. Francisco, T. D. Crawford, and T. J. Lee, J. Chem. Phys. 135, 214303 (2011).

${ }^{13}$ M. Mladenović, J. Chem. Phys. 112, 1070 (2000).

${ }^{14}$ I. M. Mills, "Vibration-rotation structure in asymmetric and symmetric top molecules," in Molecular Spectroscopy-Modern Research, edited by K. N. Rao and C. W. Matthews (Academic, New York, 1972), Vol. 3, p. 115.

${ }^{15}$ M. Mladenović, Spectrochim. Acta, Part A 58, 809 (2002).

${ }^{16}$ J. M. Bowman, S. Carter, and X. Huang, Int. Rev. Phys. Chem. 22, 533 (2003).

${ }^{17}$ M. Mladenović, Spectrochim. Acta, Part A 58, 795 (2002).

${ }^{18}$ C. E. Dateo, T. J. Lee, and D. W. Schwenke, J. Chem. Phys. 101, 5853 (1994).

${ }^{19}$ J. M. Bowman, K. Christoffel, and G. Weinberg, J. Mol. Struct. (THEOCHEM) 461-462, 71 (1999).

${ }^{20}$ M. Mladenović, M. Lewerenz, M. C. McCarthy, and P. Thaddeus, J. Chem. Phys. 131, 174308 (2009).

${ }^{21}$ M. Mladenović, M. Elhiyani, and M. Lewerenz, J. Chem. Phys. 130, 154109 (2009).

${ }^{22}$ P. Botschwina, Mol. Phys. 103, 1441 (2005).

${ }^{23}$ M. Mladenović, C. Krekeler, and P. Botschwina, "Spectroscopic properties of the HOCO radical from a theoretical RCCSD(T) investigation" (unpublished). 